\title{
INCREASED LEVELS OF HEXACOSANOIC ACID IN THE BRAIN OF WISTAR RATS: A BEHAVIORAL STUDY
}

\author{
Desirèe P. Marchetti ${ }^{1}$, Daniella de M. Coelho², \\ Adriana S. Coitinho ${ }^{3,4,5}$, Edson F. M. Guzzo ${ }^{4}$, Rafael B. Padilha ${ }^{3}$, \\ Gabriel de L. Rosa ${ }^{3}$, Carmen R. Vargas ${ }^{1,2}$
}

\begin{abstract}
Introduction: X-linked adrenoleukodystrophy (X-ALD) is a peroxisomal metabolic disorder associated with mutations in the ATP-binding cassette sub-family D member1 $(A B C D 1)$ gene. Practically all male patients with X-ALD develop adrenocortical insufficiency during childhood and progressive myelopathy and peripheral neuropathy in adulthood. However, some male patients develop a fatal cerebral demyelinating disease named cerebral adrenoleukodystrophy. Although the exact mechanisms underlying brain damage in X-ALD are still poorly elucidated, it is known that hexacosanoic acid (C26:0) accumulation represents a hallmark in the pathogenesis of this disease. In this study, we examined whether an overload of C26:0 injected in Wistar rats was capable of causing behavioral changes in these animals.
\end{abstract}

Methods: Egg lecithin in ethanol was dried under a nitrogen stream and mixed with C26:0 methyl ester. Male Wistar rats at 2-3 weeks of age were obtained from Universidade Federal do Rio Grande do Sul (UFRGS), divided into 8 groups, and submitted to an open field test. We then analyzed line crossings (locomotion and exploration), rearing (orienting and investigatory responses), grooming (anxiety manifestation), and latency to move for each animal.

Results: Animals subjected to $\mathrm{C} 26: 0$ administration presented fewer crossings and rearing episodes and a higher latency to move 45 minutes after C26:0 injection. The present work yields experimental evidence that $\mathrm{C} 26: 0$, the main accumulated metabolite in X-ALD, can cause behavioral alterations in rats such as the impairment of locomotion and exploratory capabilities, as well as a reduction in orienting and investigatory responses.

Conclusion: Although our results are preliminary, they are extremely important for future studies that investigate C26:0 accumulation and locomotor impairment in patients with X-ALD.

Keywords: X-linked adrenoleukodystrophy; C26:0; Wistar rats; open field

\section{INTRODUCTION}

X-linked adrenoleukodystrophy (X-ALD) is a progressive neurodegenerative disease with an estimated incidence of 1 in 17000 that has been diagnosed in all geographic regions and ethnic groups ${ }^{1}$. This disease is caused by mutations in the $A B C D 1$ gene; this gene encodes the peroxisomal membrane protein ALDP, which is involved in the transmembrane transport of very long-chain fatty acids (VLCFA) into peroxisomes ${ }^{2}$. As a consequence of this mutation, VLCFAs (mainly tetracosanoic acid [C24:0] and hexacosanoic acid [C26:0]) accumulate in the plasma and tissues, including the white matter of the brain, spinal cord, and adrenal cortex ${ }^{3,4}$.
1 Programa de Pós-Graduação em Ciências Biológicas - Bioquímica, Instituto de Ciências Básicas da Saúde, Universidade Federal do Rio Grande do Sul (UFRGS). Porto Alegre, RS, Brasil.

2 Serviço de Genética Médica, Hospital de Clínicas de Porto Alegre (HCPA). Porto Alegre, RS, Brasil.

3 Departamento de Microbiologia, Imunologia e Parasitologia, Instituto de Ciências Básicas da Saúde, Universidade Federal do Rio Grande do Sul (UFRGS). Porto Alegre, RS, Brasil.

4 Programa de Pós-Graduação em Farmacologia e Terapêutica, Instituto de Ciências Básicas da Saúde, Universidade Federal do Rio Grande do Sul (UFRGS). Porto Alegre, RS, Brasil.

5 Programa de Pós-Graduação em Ciências Biológicas - Fisiologia, Instituto de Ciências Básicas da Saúde, Universidade Federal do Rio Grande do Sul (UFRGS). Porto Alegre, RS, Brasil.

Corresponding author: Desirèe Padilha Marchetti desireepmarchetti@gmail.com Serviço de Genética Médica, Hospital de Clínicas de Porto Alegre Rua Ramiro Barcelos, 2350 90035-003, Porto Alegre, RS, Brasil. 
Marchetti et al.

The disease presents great phenotypic variability, ranging from the rapidly progressive cerebral form that leads to total disability within the first decade to the milder adrenomyeloneuropathy (AMN), which is compatible with survival until the eighth decade after disease onset. The literature describes 7 main phenotypes in hemizygous male patients: the childhood cerebral form (CCER), juvenile cerebral form, adult cerebral form, AMN, Addison's only, olivopontocerebellar, and asymptomatic forms ${ }^{5}$.

The cerebral form is the most progressive, severe, and devastating phenotype of this disease, and CCER is the most frequent form, with an insidious onset. These patients seem unaffected until the age of 2-10 years, when adrenal insufficiency, progressive neurological dysfunction (including problems with walking and locomotion), and cognitive deficits involving visuospatial and visuomotor functions, as well as attention and reasoning, start to develop. Symptoms depend on the site of the initial lesions. In elementary school-aged boys, the first symptoms are usually cognitive deficits and behavioral problems manifesting as a decline in school performance ${ }^{1}$. These early clinical symptoms are often misdiagnosed as attention deficit hyperactivity disorder and can delay the diagnosis of CCER. Other symptoms start to appear as the disease progresses, such as withdrawn or hyperactive behavior, apraxia, astereognosis, hearing impairment, decreased visual acuity, hemiparesis or spastic tetraparesis, cerebellar ataxia, and seizures. Death usually occurs 2 to 4 years after symptom onset ${ }^{5,6}$.

"Pure" AMN (without cerebral involvement) is a noninflammatory distal axonopathy that involves the long tracts of the spinal cord and, to a lesser extent, the peripheral nerves. Patients present paraparesis, sphincter disturbances, sensory changes, incoordination, pain, and impotence. The age of onset of AMN varies between the second and the fourth decade of life, and cerebral changes develop in approximately half of the patients. Patients with cerebral AMN can present dementia, behavioral disturbances, psychosis, epilepsy, aphasia, visual loss, and bulbar palsy ${ }^{7}$. Patients with the Addison's only form of the disease $(10 \%$ to $20 \%$ of X-ALD patients) have primary adrenal insufficiency without evidence of nervous system involvement, but are at high risk of eventually developing AMN. Some patients with the genetic defect are free of adrenal insufficiency and neurologic disabilities despite their high levels of saturated VLCFA; these are classified as asymptomatic and present a high risk of developing the CCER phenotype ${ }^{8}$.

Our research group observed changes in glial cell metabolism with the addition of C26:0 in cell culture ${ }^{9}$, but the behavioral effect of the administration of $\mathrm{C} 26: 0$ in rats has not yet been described in the literature.
Therefore, we aimed to investigate whether an overload of C26:0, achieved through intraperitoneal injection in wild type Wistar rats, causes behavioral changes in these animals.

\section{METHODS}

\section{Vesicle preparation}

Ethanol containing $8 \mathrm{mg}$ of egg lecithin (SigmaAldrich, St. Louis, USA) was dried under a nitrogen stream and mixed with $4 \mathrm{mg}$ of C26:0 methyl ester (Sigma-Aldrich). The sample was suspended in $2.5 \mathrm{~mL}$ of sterile water and then sonicated with a microtip (Sonicator Ultrasonic Processor XL - Misonix Inc., Famingdale, USA). The same procedure, without the C26:0 methyl ester, was performed to obtain pure lecithin vesicles used as the vehicle control ${ }^{10,11}$.

\section{Animals}

Male Wistar rats were obtained from the central vivarium at Universidade Federal do Rio Grande do Sul (UFRGS) at 2-3 weeks of age. The animals were immediately housed in polypropylene boxes measuring $41 \times 34 \times 16 \mathrm{~cm}$ (up to 4 animals per cage) and kept in a 12 hour light-dark environment at $23 \pm 1^{\circ} \mathrm{C}$. The animals had ad libitum access to pelleted chow and water. The experiments were performed in accordance with the Guide for the Care and Use of Laboratory Animals, DHEW Publication No. (NIH) 80-23 (1985) and were approved by the local Ethics Committee at UFRGS.

\section{Experimental groups}

The animals were divided into 8 experimental groups (6 animals in each group): Basal control (without injection); saline control (received $100 \mu \mathrm{L}$ of $0.9 \%$ sodium chloride); lecithin vesicle $15 \mathrm{~min}$, $45 \mathrm{~min}$, and $60 \mathrm{~min}$ (received $100 \mu \mathrm{L}$ of lecithin vesicle and were sacrificed 15,45 , or 60 minutes after injection, respectively); vesicle + C26 $15 \mathrm{~min}, 45 \mathrm{~min}$, and 60 min (received $100 \mu \mathrm{L}$ of vesicles with C26:0 methyl ester at a final concentration of $4 \mathrm{mM}$ and were sacrificed 15,45 , or 60 minutes after injection, respectively). All treatments were administered by intraperitoneal injection.

\section{Behavioral test}

Rats were submitted to a behavioral task using an open field test and sacrificed 5 minutes later. The test used an acrylic box whose bottom was divided by black lines into 12 equisized quadrants; animals were placed on one specific quadrant, facing the box wall, and were left to freely explore the box for 5 minutes. The open field apparatus was a $40 \mathrm{~cm} \times 60 \mathrm{~cm}$ linoleum floor area surrounded on three sides by a $60 \mathrm{~cm}$ plywood wall and on the other, by a $60 \mathrm{~cm}$ glass wall. 
Line crossings (locomotion and exploration), rearing (orienting and investigatory responses), grooming (anxiety manifestation), and latency to move were analyzed for each rat ${ }^{12}$. After each test, the floor was cleaned with water and $70 \%$ ethanol to avoid a potential excitatory effect produced by urine or fecal residues.

\section{Brain microdissection and tissue preparation}

At the end of the study, the animals were sacrificed by decapitation and the brains were immediately removed and placed on a cold plate. The whole brains were dissected and chilled until homogenization in 1:5 (w/v) saline solution $(0.9 \% \mathrm{NaCl})$. The homogenate was centrifuged at $800 \times \mathrm{g}$ for $10 \mathrm{~min}$ at $4^{\circ} \mathrm{C}$, and the supernatant was used for determining $\mathrm{C} 26: 0$ concentrations.

\section{Fatty acid analysis}

C26:0 was analyzed according to Moser and Moser $^{13}$. The procedure consisted in the preparation of a total lipid extract, which was treated with methanolic $\mathrm{HCl}(3 \mathrm{~N})$ for the production of fatty acid methyl esters; these were then purified by thin-layer chromatography. The purified esters were extracted with hexane and analyzed by gas chromatography. We used a Varian gas chromatographer (Agilent Technologies, Santa Clara, USA) with an HP-5 column ( $5 \%$ methylphenyl silicone, $0.33 \mathrm{~mm}$ film thickness, $0.2 \mathrm{~mm}$ inner diameter and $25 \mathrm{~m}$ column length), a flame ionization detector, a split/splitless injector, and helium as the mobile phase. C26:0 concentrations were expressed in $\mu \mathrm{mol} / \mathrm{L}$. Heptacosanoic acid was used as an internal standard.

\section{Statistical analysis}

Results were expressed as mean \pm standard error of the mean (SEM). Comparisons between mean values were made using one-way analysis of variance (ANOVA), followed by a Tukey's post-hoc test. p values $<0.05$ were considered significant. GraphPad Prism version 5.0 (GraphPad Software Inc., San Diego, USA) was used for all statistical analyses and graphs.

\section{RESULTS}

\section{Whole-brain C26:0 concentration}

Figure 1 shows the concentrations of $\mathrm{C} 26: 0$ in the whole brains of rats in each of the 8 studied groups. Rats that received an intraperitoneal injection of C26:0 in lecithin vesicles and were sacrificed after 45 minutes presented higher brain C26:0 levels when compared to the other groups $(F[7,40]=8.08, p<0.0001)$.

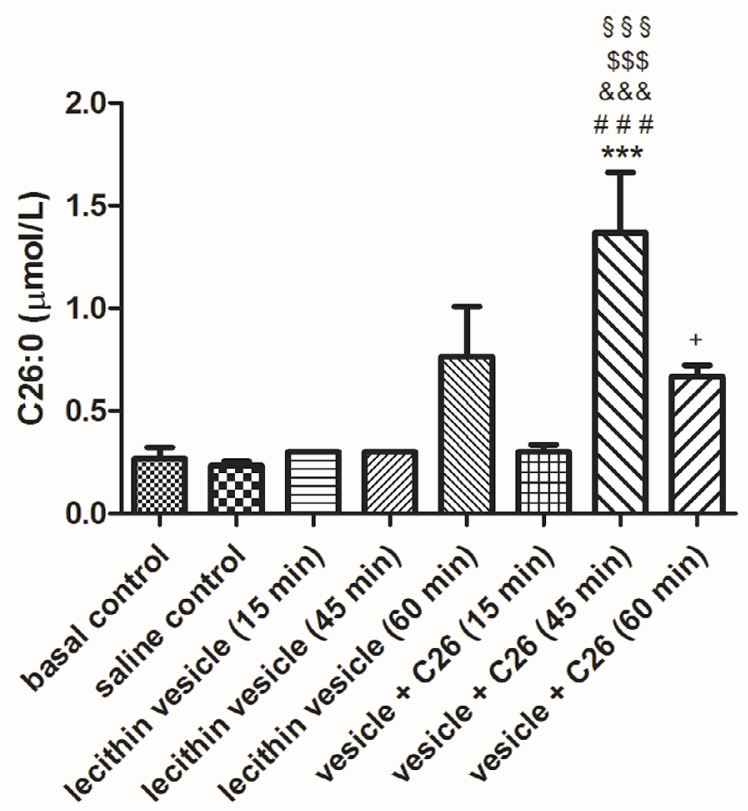

Figure 1: C26:0 concentrations in the rats' whole brains. Results represent mean \pm standard error of the mean (SEM). $" x p<0.001$ compared to the basal control group. ${ }^{\# \# p} p 0.001$ compared to the saline control group. \& \& \& $p<0.001$ compared to the lecithin vesicle (15 min) group. ${ }^{\$ \$ \$} p<0.001$ compared to the lecithin vesicle $(45 \mathrm{~min})$ group. $\$ \$ \$ p<0.001$ compared to the vesicle + C26 (15 min) group. ${ }^{+} p<0.05$ compared to the vesicle + C26 (45 min) group. One-way analysis of variance (ANOVA), followed by a Tukey's post-hoc test.

\section{Behavioral tests}

Results of the open field task are displayed in Figure 2. Figure 2a shows reduced locomotion and exploratory capabilities (represented by fewer crossings) in rats that received the $\mathrm{C} 26: 0$ and lecithin vesicle injection and were sacrificed after 45 minutes when compared to other groups $(F[7,40]=10.74$, $p<0.0001$ ]. Figure $2 b$ corroborates these results by showing that the same animals presented a higher latency to initiate locomotion (expressed in seconds) compared to other groups $(F[7,38]=6.59, p<0.0001]$. Similarly, Figure $2 c$ shows that rats belonging to this group presented reduced orienting and investigatory responses (measured by the number of rearing episodes $)(F[7,40]=5.41, p<0.0001)$. Figure $2 d$ illustrates the number of anxiety manifestations (grooming); no significant differences were observed in this behavioral aspect among the 8 studied groups $(F[7,40]=0.67, p>0.05)$. 

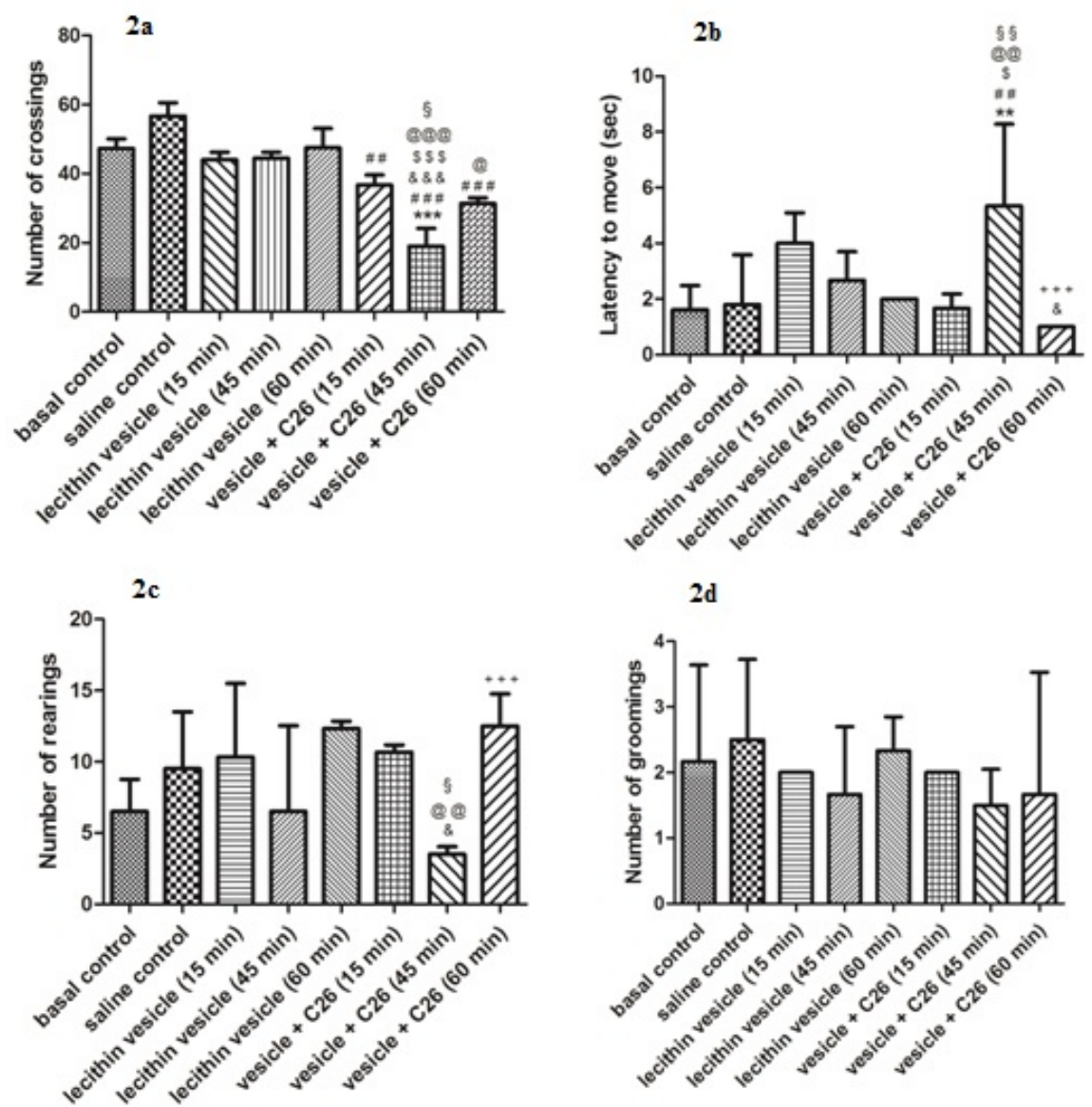

Figure 2: Open field tasks. A: Crossings; B: Latency to initiate locomotion; C: Rearing; D: Grooming.

${ }^{* *} p<0.01$ and ${ }^{* * *} p<0.001$ compared to the basal control group. ${ }^{\#} p<0.01$ and ${ }^{\# \#} p<0.001$ compared to the saline control group. ${ }^{\circledR} p<0.05$ and \& \& \&p<0.001 compared to the lecithin vesicle (15 min) group. ${ }^{\$} p<0.05$ and $\$ \$ \$ p<0.001$ compared to the lecithin vesicle (45 min) group. $@ p<0.05$, @ $p<0.01$, and @@@ $p<0.001$ compared to the lecithin vesicle $(60 \mathrm{~min})$ group. ${ } s<0.05$ and $\$ \$ p<0.01$ compared to the vesicle + C26 (15 min) group. ${ }^{++} p<0.001$ compared to the vesicle + C26 (45 min) group. Results represent mean \pm standard error of the mean (SEM). One-way analysis of variance (ANOVA), followed by a Tukey's post-hoc test.

\section{DISCUSSION}

$\mathrm{X}-\mathrm{ALD}$ is an inborn error of the peroxisome metabolism characterized by a progressive demyelination of the brain's white matter and adrenal insufficiency. Mutations in the $A B C D 1$ gene result in several phenotypic variants that vary in aspects such as age of onset, rate of disease progression, and primary affected tissues. Although the mechanisms related to tissue damage are poorly understood, it is believed that the main accumulated metabolite (C26:0) could contribute, at least in part, to the pathophysiology of this disease. In this work, we investigated whether excess C26:0, administrated by intraperitoneal injection, might induce behavioral changes in male Wistar rats.

Here, we presented compelling evidence that a lecithin vesicle enriched with $\mathrm{C} 26: 0$ was able to reach the brain, and that a peak in C26:0 concentration was verified in rats sacrificed 45 minutes after injection in comparison to the other groups. Figure 1 shows that C26:0 concentration is significantly higher in the "vesicle + C26" group sacrificed 45 minutes after injection when compared to the other groups, especially considering those animals sacrificed after 15 or 60 minutes. In addition, C26:0 concentrations in animals sacrificed after 1 hour decreased again and were similar to the other groups, illustrating the kinetics of acid concentration. This probably occurred because the fatty acid was oxidized inside the peroxisome, since the rats used in this study were not knockouts and did not have a dysfunction in the $A B C D 1$ gene. According to the literature, surprisingly, no detectable elevations in plasmatic concentrations of C24:0 or C26:0 were detected in $X$-ALD knockout mice, and this was in line with our results (data not shown) ${ }^{14}$. Furthermore, the same authors reported a 5-fold increase in C26:0 levels in the brain of knockout rats compared to other tissues. In this work, we verified that Wistar rats that received an intraperitoneal administration of C26:0 and were 
sacrificed after 45 minutes presented a mean brain C26:0 level of $1.37 \mu \mathrm{mol} / \mathrm{L}$, which would approach plasmatic levels of male X-ALD patients. In humans, $X-A L D$ is usually diagnosed by measuring VLCFA levels in plasma ${ }^{13}$; male patients present a mean range of 1.36-4.94 $\mu \mathrm{mol} / \mathrm{L}$ of $\mathrm{C} 26: 0$, female heterozygotes have a mean range of $1.41-2.33 \mu \mathrm{mol} / \mathrm{L}$, and healthy individuals present a mean range of $0.11-0.96 \mu \mathrm{mol} / \mathrm{L}^{15}$.

Behavioral tests are important in biomedical research for testing novel treatments, investigating currently used drugs, and in mechanistic studies. The open field task is one of the most commonly used tests for measuring animal behaviors. It is a fast and relatively easy test that provides a variety of behavioral information ranging from the subject animal's general ambulatory ability to its emotionality ${ }^{16}$. We performed the open field test and assessed the number of crossings and grooming and rearing episodes, and measured the latency to locomotion. Results indicated that Wistar rats injected with C26:0 developed an acute impairment of the central nervous system, since they presented a delay in initiating locomotion, decreased exploratory capabilities, and reduced orienting and investigatory responses.

It is important to highlight that these changes were observed in rats sacrificed 45 minutes after C26:0 injection, suggesting that these results express the kinetics of acid degradation since the effects were still not pronounced in rats sacrificed after 15 minutes. Figure 2a illustrates that rats injected with $\mathrm{C} 26: 0$ and sacrificed after 45 minutes performed fewer crossings when compared to the control groups and to the group that received lecithin injection and was sacrificed after 45 minutes. Similarly, when observing latency to initiate locomotion, we noted that animals belonging to the "vesicle + C26 - 45 min" group presented a higher latency time to move when compared to the control groups and to the group that received lecithin alone and was sacrificed after 45 minutes. By analyzing these two tests, we can clearly note the exclusive effect of $\mathrm{C} 26: 0$ on the rats behavior and also affirm that lecithin (the vehicle component of the vesicles) had no influence on the results. Considering the rearing episodes, Figure $2 c$ shows that rats in the "vesicle + C26 - 45 min" group presented fewer events when compared to the "lecithin vesicle - $15 \mathrm{~min}$," "lecithin vesicle - $60 \mathrm{~min}$," and "vesicle + C26 - 15 min" groups. When analyzing the test results of animals in the "vesicle + C26 - 60 min" group, it is possible to infer that the acid had already been oxidized 1 hour after the injection, because the behavior tended to be restored in comparison to the "vesicle + C26 - 45 min" group. Moreover, Figure $2 \mathrm{~d}$ (number of grooming episodes) shows that no significant differences were observed among the 8 studied groups; that is, the rats presented no differences in anxiety manifestations.

The work performed by Pujol and colleagues ${ }^{17}$ corroborated our results concerning behavioral alterations. Their study has shown that older $A L D$ deficient mice (knockout) exhibited an abnormal neurological and behavioral phenotype, starting at approximately 15 months of age. Additionally, $\mathrm{X}$ - $A L D$-mutant rats at 20 months of age showed severe impairment in motor coordination abilities (verified by a rotarod test) and presented a significant reduction in exploratory activities or hypoactivity during an open field test. The authors related these behavioral effects with a slower nerve conduction and with myelin and axonal anomalies detected in the spinal cord and sciatic nerve, which correspond to what is observed in patients with AMN.

However, as opposed to the work performed by Pujol and colleagues ${ }^{17}$, the rats used in our work were young (2-3 weeks of age) and had not yet reached sexual maturity. We might presume that the administration of C26:0 in healthy rats induces behavioral changes that are similar to those of a child presenting the initial symptoms of the disease, considering that the earliest CCER symptoms frequently include cognitive dysfunction manifested as behavioral disturbances. In order to further complement our work, more studies would be needed to confirm our hypothesis; for instance, a neuroimaging study of the Wistar rats' brains, or increased numbers of C26:0 injections and time periods between injection and sacrifice for simulating disease chronicity.

Altogether, our data demonstrates for the first time in the literature that $\mathrm{C} 26: 0$, by itself, can induce behavioral alterations in young Wistar rats at the motor capacity level, in an acute time of 45 minutes after C26:0 injection. These results are preliminary, but extremely important and promising for future studies of X-ALD pathophysiology and for understanding the relationship between C26:0 accumulation and locomotor impairment in human patients.

\section{Conflicts of Interest}

The authors declare that they have no conflicts of interest.

\section{Acknowledgments}

This study was supported by Coordenação de Aperfeiçoamento de Pessoal de Nível Superior (CAPES), Conselho Nacional de Desenvolvimento Científico e Tecnológico (CNPq), and Fundo de Incentivo à Pesquisa e Eventos (FIPE/HCPA). 


\section{REFERENCES}

1. Kemp S, Huffnagel IC, Linthorst GE, Wanders RJ, Engelen M. Adrenoleukodystrophy: neuroendocrine pathogenesis and redefinition of natural history. Nat Rev Endocrinol. 2016;12(10):606-15.

2. Moser HW. Adrenoleukodystrophy: phenotype, genetics, pathogenesis and therapy. Brain. 1997; 120(8):1485-508.

3. Moser HW, Smith KD, Watkins PA, Powers J, Moser AB. X-linked adrenoleukodystrophy. In: Scriver CR, Beaudet al, Sly WS, Valle D, editors. The metabolic and molecular bases of inherited disease. 8th ed. New York: McGraw-Hill, Inc; 2001.

4. Moser A, Jones R, Hubbard W, Tortorelli S, Orsini J, Caggana M, Vogel B, Raymond G. Newborn screening for $X$-linked adrenoleukodystrophy. Int J Neonatal Screen. 2016;2(4):15.

5. Engelen M, Kemp S, Visser M, Geel BM, Wanders RJA, Aubourg P, Poll-The BT. X-linked adrenoleukodystrophy (X-ALD): clinical presentation and guidelines for diagnosis, follow-up and management. Orphanet J Rare Dis. 2012;7(1):51.

6. Engelen M, Kemp S, Poll-The BT. $X$-linked adrenoleukodystrophy: pathogenesis and treatment. Curr Neurol Neurosci Rep. 2014;14(10):486.
7. Moser HW, Raymond GV, Dubey P. Adrenoleukodystrophy: new approaches to a neurodegenerative disease. JAMA. 2005;294(24):3131-4.

8. Berger J, Gärtner J. X-linked adrenoleukodystrophy: clinical, biochemical and pathogenetic aspects. Biochim Biophys Acta. 2006;1763(12):1721-32.

9. Marchetti DP, Steffens L, Jacques CE, Guerreiro GB, Mescka CP, Deon M, et al. Oxidative imbalance, nitrative stress, and inflammation in c6 glial cells exposed to hexacosanoic acid: protective effect of $\mathrm{N}$-acetyl-L-cysteine, trolox, and rosuvastatin. Cell Mol Neurobiol. 2018;38(8):1505-16.

10. Di Biase A, Di Benedetto R, Fiorentini C, Travaglione S, Salvati S, Attorri $\mathrm{L}$, et al. Free radical release in $\mathrm{C} 6$ glial cells enriched in hexacosenoic acid: implication for $\mathrm{X}$-linked adrenoleukodystrophy pathogenesis. Neurochem Int. 2004;44(4):215-21.

11. Di Biase A, Benedetto R, Salvati S, Attorri L, Leonardi F, Pietraforte D. Effects of L-mono methyl-arginine, $\mathrm{N}$-acetyl- cysteine and diphenyleniodonium on free radical release in $\mathrm{C} 6$ glial cells enriched in hexacosanoic acid. Neurochem Res. 2005;30(2):215-23.
12. Izquierdo I. Different forms of posttraining memory processing. Behav Neural Biol. 1989;51(2):171-202.

13. Moser HW, Moser AB. Measurement of saturated very long chain fattyacid in plasma. In: Hommes FA, editor. Techniques of diagnostic human biochemical genetics. New York: Wiley-Liss; 1991. p. 177-91.

14. Lu JF, Lawler AM, Watkins PA, Powers JM, Moser AB, Moser HW, et al. A mouse model for $X$-linked adrenoleukodystrophy. Proc Natl Acad Sci U S A. 1997;94(17):9366-71.

15. Marchetti DP, Donida B, Jacques CE, Deon M, Hauschild TC, KoehlerSantos $P$, et al. Inflammatory profile in X-linked adrenoleukodystrophy patients: understanding disease progression. J Cell Biochem. 2018;119(1):1223-33.

16. Walsh RN, Cummins RA. The openfield test: a critical review. Psychol Bull. 1976;83(3):482-504.

17. Pujol A, Hindelang C, Callizot N, Bartsch U, Schachner M, Mandel JL. Late onset neurological phenotype of the X-ALD gene inactivation in mice: a mouse model for adrenomyeloneuropathy. Hum Mol Genet. 2002;11(5):499-505.

Received: May 23, 2020 Accepted: Oct 11, 2020 